\title{
Radiation Transport Around Axisymmetric Blunt Body Vehicles Using a Modified Differential Approximation
}

\author{
Lin C. Hartung* \\ NASA Langley Research Center, Hampton, VA 23665-5225 \\ and H. A. Hassan ${ }^{\dagger}$ \\ North Carolina State University, Raleigh, NC 27695
}

\begin{abstract}
A moment method for computing 3 -D radiative transport is applied to axisymmetric flows in thermochemical nonequilibrium. Such flows are representative of proposed aerobrake missions. The method uses the P-1 approximation to reduce the governing system of integro-differential equations to a coupled set of partial differential equations. A numerical solution method for these equations given actual variations of the radiation properties in thermochemical nonequilibrium blunt body flows is developed. Initial results from the method are shown and compared to tangent slab calculations. The agreement between the transport methods is found to be about 10 percent in the stagnation region, with the difference increasing along the flank of the vehicle.

*Aerospace Engineer, Aerothermodynamics Branch, Space Systems Division, Member AIAA.

†Professor, Department of Mechanical and Aerospace Engineering, Associate Fellow AIAA.

Received Nov. 11, 1991; presented as Paper 92-0119 at the AIAA 30th Aerospace Sciences Meeting, Reno, NV, Jan. 6-9, 1992; revision received March 8, 1992; accepted for publication March $12,1992$. Copyright (c) 1992 by the American Institute of Aeronautics and Astronautics, Inc. No copyright is asserted in the United States under Title 17, U.S. Code. The U.S Government has a royalty-free license to exercise all rights under the copyright claimed herein for Governmental purposes. All other rights are reserved by the copyright owner.
\end{abstract}




\section{Nomenclature}

\begin{tabular}{|c|c|}
\hline$a$ & Grid cell surface area $\left(\mathrm{cm}^{2}\right)$ \\
\hline$B_{\nu}$ & Radiosity $\left(\mathrm{W} / \mathrm{cm}^{2}-\mathrm{s}^{-1}\right)$ \\
\hline$C$ & Constant in time term \\
\hline$d$ & Coefficients in Gauss-Seidel formulation \\
\hline$G_{\nu}$ & Incident radiative intensity $\left(\mathrm{W} / \mathrm{cm}^{2}-\mathrm{s}^{-1}\right)$ \\
\hline$I_{\nu}$ & Radiative intensity (W/ $\mathrm{cm}^{2}-\mathrm{s}^{-1}$-ster) \\
\hline$j_{\nu}^{e}$ & Emission coefficient $\left(\mathrm{W} / \mathrm{cm}^{3}{ }_{-}{ }^{-1}-\right.$ ster $)$ \\
\hline$L$ & Local error in Gauss-Seidel iteration \\
\hline$\hat{n}$ & Surface unit normal \\
\hline$\vec{q}_{\mathrm{R}}$ & Radiative flux $\left(\mathrm{W} / \mathrm{cm}^{2}\right)$ \\
\hline$r$ & Relaxation parameter \\
\hline$\vec{r}$ & Position vector \\
\hline$R^{n}$ & Residual of Eq. 18 \\
\hline$s$ & Path variable $(\mathrm{cm})$ \\
\hline$T$ & Temperature (K) \\
\hline$V$ & Volume of grid cell $\left(\mathrm{cm}^{3}\right)$ \\
\hline$x$ & Cartesian coordinate $(\mathrm{cm})$ \\
\hline$y$ & Cartesian coordinate $(\mathrm{cm})$ \\
\hline$z$ & Cartesian coordinate $(\mathrm{cm})$ \\
\hline , & Computational variable for incident intensity \\
\hline$\varepsilon$ & Surface emissivity \\
\hline$\zeta$ & Third computational coordinate direction \\
\hline$\eta$ & Second computational coordinate direction \\
\hline$\kappa^{\prime}$ & Absorption coefficient corrected for induced emission $(1 / \mathrm{cm})$ \\
\hline$\mu$ & Cosine of angle between $\hat{\Omega}$ and $z$-axis \\
\hline$\nu$ & Frequency $(1 / \mathrm{s})$ \\
\hline
\end{tabular}


$\xi \quad$ First computational coordinate direction

$\tau \quad$ Optical variable

$\omega \quad$ Solid angle (steradians)

$\hat{\Omega} \quad$ Direction vector

\section{Sub- and Superscripts}

$\begin{array}{ll}i & \text { grid index in } \mathrm{x} \text { or } \xi \text {-direction } \\ I & \text { cell center index in } \mathrm{x} \text { or } \xi \text {-direction } \\ j & \text { grid index in y or } \eta \text {-direction } \\ J & \text { cell center index in y or } \eta \text {-direction } \\ k & \text { grid index in z or } \zeta \text {-direction } \\ K & \text { cell center index in z or } \zeta \text {-direction } \\ m & \text { medium } \\ p & \text { Planck } \\ \mathrm{R} & \text { radiative } \\ w & \text { wall } \\ \nu & \text { spectral } \\ & \end{array}$

\section{Introduction}

Figure 1 shows some important features of an aerobrake flowfield in a planetary atmosphere at the high speed typical of interplanetary flight. A significant portion of the heating experienced by such a blunt spacecraft can be due to radiation. The radiative heating can therefore be a strong driver on the design of the spacecraft heatshield. This heating is predicted in two steps: first the radiative properties are computed at each point in the flowfield; 
then the transport of radiation to the body surface is calculated. The first part of the problem, for a flowfield including the features of Fig. 1, was treated in Ref. 1. For the radiative transport calculation the traditional model in a hypersonic, blunt body flow is the tangent slab or plane parallel approximation. ${ }^{2-8}$ To further simplify the transport calculation, this model is sometimes applied to the visible and infrared region only, with absorption effects ignored. 9,10

Recent work in aerothermodynamics ${ }^{9}$ has provided some evidence that the $1 \mathrm{D}$ tangent slab approximation may not be adequate even at the stagnation point for some of the vehicle shapes and applications under consideration for future space missions. It certainly will not suffice to compute radiative transport in the wake region where it is planned to locate unshielded payloads. No 3-D transport algorithm currently in use for flowfield solutions has been identified, though an effort toward this end is underway by Edwards et al. ${ }^{11}$.

The objective of the present work is to initiate the development of a 3 - $\mathrm{D}$ transport algorithm for radiative transfer in blunt body flowfields. This initial work is limited to axisymmetric forebody flowfields and does not include the effects of flowfield coupling. The geometry is a deformed pie slice, with a cross-section like that shown in Fig. 2. Since an exact solution of the radiative transport equations in 3 - $\mathrm{D}$ media is not feasible because it requires solving an excessive number of equations, an approximate method is used. The moment method is chosen here, reducing the problem from integro-differential equations to simpler partial differential equations. This method was originally developed for 1-D radiative transport, but recent work has extended it to 2- and 3-D geometries with various restrictions (see Ref. 12 and past work cited there). This paper discusses the application of the method to the current problem, including required changes for nonequilibrium, and presents some initial results.

\section{Radiative Transport Theory}

The complete equation of radiative transport has been derived in many standard texts. 
Neglecting transients and assuming a non-scattering medium it becomes:

$$
\frac{d I_{\nu}(s, \hat{\Omega})}{d s}+\kappa_{\nu}^{\prime}(s) I_{\nu}(s, \hat{\Omega})=j_{\nu}^{e}(s)
$$

Since for some frequencies $\kappa_{\nu}^{\prime}$ can be negative in part of a nonequilibrium flow, ${ }^{1}$ it will pass through zero somewhere in the domain. The usual procedure of dividing by $\kappa_{\nu}^{\prime}$ to transform this equation to an optical variable would therefore introduce a singularity. For this work, then, Eq. 1 will be left in terms of the physical coordinate.

Equation 1 applies at each frequency, $\nu$, and for each direction, $\hat{\Omega}$. A formal solution can be written using an integrating factor. The radiative transport can then be computed numerically for a finite number of frequency points and directions. Some method of selecting appropriate frequencies at which to compute the radiative properties $j_{\nu}^{e}$ and $\kappa_{\nu}^{\prime}$ in a nonequilibrium flow must be devised. That problem was discussed in Ref. 1. For the cases in this paper, 1066 optimized frequency points were used to compute the radiation occurring between 0.31 and $16.5 \mathrm{eV}$.

\section{Plane-Parallel Medium}

A common approximation for solving the radiative transport equation is to assume that the properties of the medium through which the radiation travels vary in only one direction. This situation is illustrated by the inset in Fig. 2. It may be a reasonable assumption in the stagnation region of a flowfield, where gradients are mainly in the normal direction. In this case $s$ and $\hat{\Omega}$ are replaced by $z$ and $\mu$ (the cosine of the angle between $\hat{\Omega}$ and the $z$-axis). The derivative $d / d s$ then becomes $\mu \partial / \partial z$. To accommodate the boundary conditions in this case, $I$ is split into forward $(\mu>0)$ and backward $(\mu \leq 0)$ components. The formal solution for each can be written:

$$
\begin{aligned}
& I_{\nu}^{+}(z, \mu)=I_{\nu}^{+}(0, \mu) \exp \left[-\int_{0}^{z} \kappa_{\nu}^{\prime}\left(s^{\prime \prime}, \mu\right) / \mu d s^{\prime \prime}\right] \\
& +\int_{0}^{z} j_{\nu}^{e}\left(s^{\prime}, \mu\right) / \mu \exp \left[-\int_{s^{\prime}}^{z} \kappa_{\nu}^{\prime}\left(s^{\prime \prime}, \mu\right) / \mu d s^{\prime \prime}\right] d s^{\prime} \\
& I_{\nu}^{-}(z, \mu)=I_{\nu}^{-}\left(z_{0}, \mu\right) \exp \left[-\int_{z_{0}}^{z} \kappa_{\nu}^{\prime}\left(s^{\prime \prime}, \mu\right) / \mu d s^{\prime \prime}\right]
\end{aligned}
$$




$$
+\int_{z_{0}}^{z} j_{\nu}^{e}\left(s^{\prime}, \mu\right) / \mu \exp \left[-\int_{s^{\prime}}^{z} \kappa_{\nu}^{\prime}\left(s^{\prime \prime}, \mu\right) / \mu d s^{\prime \prime}\right] d s^{\prime}
$$

The $z=0$ boundary is the vehicle surface, which is assumed to emit radiation according to Planck's function at the wall temperature $T_{w}$. The $z=z_{0}$ boundary is the freestream gas ahead of the vehicle which is assumed not to emit radiation. Along the surface normal $(\mu= \pm 1)$, and introducing the optical variable:

$$
\tau_{\nu}(z)=\int_{0}^{z} \kappa_{\nu}^{\prime}\left(s^{\prime}\right) d s^{\prime}
$$

these then become:

$$
\begin{gathered}
I_{\nu}^{+}(z, 1)=I_{\nu p}\left(T_{w}\right) e^{-\tau_{\nu}(z)}+\int_{0}^{z} j_{\nu}^{e}\left(s^{\prime}\right) e^{\tau_{\nu}\left(s^{\prime}\right)-\tau_{\nu}(z)} d s^{\prime} \\
I_{\nu}^{-}(z,-1)=\int_{z}^{z_{0}} j_{\nu}^{e}\left(s^{\prime}\right) e^{\tau_{\nu}(z)-\tau_{\nu}\left(s^{\prime}\right)} d s^{\prime}
\end{gathered}
$$

To obtain the radiative flux in an absorbing, plane parallel medium it is easy to show that, if the exponential approximation is used to replace the exponential integrals, the above equations give the radiative flux, $q_{\nu}=q_{\nu}^{+}+q_{\nu}^{-}$, when $\tau_{\nu}$ is replaced by $2 \tau_{\nu}$ and a factor of $\pi$ is added. The divergence of the radiative flux is obtained by differentiating this equation with respect to $z$, then integrating over the complete spectral range. The differentiation can be done analytically, or numerically from the solution for $q_{\nu}(z)$. The latter approach has been implemented here, following Nicolet. ${ }^{2}$ The analytical approach requires a numerical differentiation of the integrand and would be of similar accuracy.

\section{Numerical Solution}

The integrals in Eqs. 4-6 require some care. Because $\kappa_{\nu}^{\prime}$ can be negative in some regions, ${ }^{1}$ the log-linear variation assumed by Nicolet $^{2}$ in the RAD/EQUIL code cannot be used. Examination of $\kappa_{\nu}^{\prime}$ profiles suggests that a piecewise linear approximation is acceptable.

For the intensity integrals, the flowfield grid spacing may be such that for strongly absorbing spectral regions $\Delta z$ is not small with respect to variations in the integrand. To integrate accurately, examination of $\tau_{\nu}$ profiles suggests a piecewise linear approximation be 
used. Setting $\tau_{\nu}=a_{i} s+b_{i}$ in each grid interval and approximating $j_{\nu}^{e}$ by its average at the two ends of the interval, the integral over the exponential term can be carried out. (The obvious approach of also setting $j_{\nu}^{e}=c_{i} s+d_{i}$ has numerical problems for small $\tau_{\nu}$, resulting from terms which go to $0 / 0$.)

\section{Three-Dimensional Medium}

When the tangent slab approximation is invalid, the directional variation of the radiation must be accounted for. The Modified Differential Approximation (MDA) of Modest ${ }^{12}$ is reported to give results of excellent accuracy for all optical conditions by splitting the radiative intensity into medium and wall components. The smoothly varying medium intensity can then be solved using the P-1 approximation. Adapting Modest's derivation for the current non-gray, nonscattering, and nonequilibrium flow gives the following governing equation for the medium intensity (this result is equivalent to Eq. 1 when $I$ is split):

$$
\nabla \cdot\left(\hat{\Omega} I_{\nu_{m}}\right)=j_{\nu}^{e}-\kappa_{\nu}^{\prime} I_{\nu_{m}}(\vec{r}, \hat{\Omega})
$$

Taking the zeroth and first moments of this equation and integrating over $4 \pi$ steradians yields the applicable governing equations for the incident intensity, $G_{\nu}$, and radiative flux, $\vec{q}_{\nu}$, of the medium:

$$
\begin{gathered}
\nabla \cdot \vec{q}_{\nu_{m}}=4 \pi j_{\nu}^{e}-\kappa_{\nu}^{\prime} G_{\nu_{m}} \\
\nabla G_{\nu_{m}}=-3 \kappa_{\nu}^{\prime} \vec{q}_{\nu_{m}}
\end{gathered}
$$

where the P-1 approximation has been used to simplify Eq. 9.

To obtain the complete radiative transport solution, the contribution to $\vec{q}$ from the walls must also be considered. This is obtained from the following integral:

$$
\vec{q}_{\nu_{w}}=\frac{1}{\pi} \int_{4 \pi} B_{\nu}\left[\vec{r}^{\prime}(\omega)\right] e^{-\tau_{\nu}} \hat{\Omega} d \omega
$$

where the wall radiosity, $B_{\nu}$, is given by the sum of emission at the wall temperature $T_{w}$, and reflection of radiation emanating from other wall surfaces. In Modest's analysis, the wall 
reflection is assumed to be diffuse. The treatment of specularly reflecting wall surfaces adds considerably to the complexity of the problem and will not be addressed here. In any case, for a forebody flowfield the wall surface is convex so that no reflection of radiation emitted by the wall can occur (this simplification will not always apply in wake flows and so will be removed in future work on this method). Then the radiosity is just the emission from the wall:

$$
B_{\nu}(\vec{r})=\varepsilon_{\nu} \pi I_{\nu p}\left[T_{w}(\vec{r})\right]
$$

This contribution to the radiative flux must be computed for each grid cell in the medium by integration over all the wall surface elements to which it has a line of sight.

If it is further assumed that the wall is cold, or that it makes a negligible contribution, then $\vec{q}_{w} \approx 0$, and only the medium contribution need be considered. (Note that a cold black wall is assumed in many of the tangent slab transport analyses, since this gives the radiation incident on the wall. For generic studies in which the wall properties are undefined, this is a meaningful quantity for comparison.) This approximation is not unreasonable for aeroassist flowfields, since heating rates are relatively low in the upper atmosphere and the wall temperature will accordingly be relatively low during much of the flight. In fact, at the maximum wall temperature for Shuttle-type reusable thermal protection systems, the peak of the black body emission spectrum occurs at about $0.75 \mathrm{eV}$. For lower temperatures, the black body peak shifts to even lower energies. In this low energy spectral region the gas is quite transparent to radiation except in isolated atomic lines (see Fig. 3) so the wall can have little effect on the divergence of the radiative flux. In that case, $\nabla \cdot \vec{q}_{\mathrm{R}} \approx \nabla \cdot \overrightarrow{q_{m}}$ and is obtained by integrating Eq. 8 over frequency. Note that unlike the plane-parallel medium, numerical differentiation is not required here.

\section{Numerical Solution}

The Computational Fluid Dynamics (CFD) algorithm thus far used in this radiation

study is a finite volume algorithm (the LAURA code of Gnoffor ${ }^{13}$ ). To obtain a form of the 
governing equations suitable for a finite-volume solution, Eq. 8 can be substituted in the divergence of Eq. 9 to obtain:

$$
\vec{\nabla} \cdot\left(\frac{1}{\kappa_{\nu}^{\prime}} \vec{\nabla} G_{\nu}\right)=3 \kappa_{\nu}^{\prime} G_{\nu}-12 \pi j_{\nu}^{e}
$$

In the finite volume approach, this is now to be integrated over the volume of a single grid cell, $V$. By application of Gauss' theorem (the divergence theorem), the volume integral on the left-hand-side can be transformed to a surface integral. Then

$$
\iint_{a}\left(\frac{1}{\kappa_{\nu}^{\prime}} \vec{\nabla} G_{\nu} \cdot \hat{n}\right) d a=\int_{V}\left(3 \kappa_{\nu}^{\prime} G_{\nu}-12 \pi j_{\nu}^{e}\right) d V
$$

The integral on the left-hand-side is performed by assuming that the absorption coefficient $\kappa_{\nu}^{\prime}$ and the gradient of the incident intensity $\vec{\nabla} G_{\nu}$ are constant on each cell face, leaving a summation over the six faces of the cell. On the right-hand-side, values at the cell center are used. The notation for the cell geometry is that of Gnoffo ${ }^{14}$, where $I, J$, and $K$ denote cell centers, and $i, j$, and $k$ denote cell faces. Then

$$
\begin{array}{r}
{\left[\left(\frac{1}{\kappa_{\nu}^{\prime}} \vec{\nabla} G_{\nu}\right)_{i+1} \cdot \vec{a}_{i+1}-\left(\frac{1}{\kappa_{\nu}^{\prime}} \vec{\nabla} G_{\nu}\right)_{i} \cdot \vec{a}_{i}\right]_{J, K}} \\
+\left[\left(\frac{1}{\kappa_{\nu}^{\prime}} \vec{\nabla} G_{\nu}\right)_{j+1} \cdot \vec{a}_{j+1}-\left(\frac{1}{\kappa_{\nu}^{\prime}} \vec{\nabla} G_{\nu}\right)_{j} \cdot \vec{a}_{j}\right]_{I, K} \\
+\left[\left(\frac{1}{\kappa_{\nu}^{\prime}} \vec{\nabla} G_{\nu}\right)_{k+1} \cdot \vec{a}_{k+1}-\left(\frac{1}{\kappa_{\nu}^{\prime}} \vec{\nabla} G_{\nu}\right)_{k} \cdot \vec{a}_{k}\right]_{I, J} \\
=V\left(3 \kappa_{\nu}^{\prime} G_{\nu}-12 \pi j_{\nu}^{e}\right)_{I, J, K}
\end{array}
$$

The remainder of the development will be restricted to axisymmetric flows, in which the second term in square brackets is zero. Cylindrical coordinates are not used, since the eventual goal is treatment of 3D flows.

For the nongray gas found in a shock layer, the radiative properties $\kappa_{\nu}^{\prime}$ and $j_{\nu}^{e}$ vary over orders of magnitude at various locations and frequencies. To minimize the numerical difficulties that this variation can introduce, some kind of normalization is desired. Define

$$
\bar{j}_{\nu}=\frac{\sum_{\text {cells }} j_{\nu}^{e}}{N_{\text {cells }}}
$$


and

$$
\bar{\kappa}_{\nu}=\frac{\sum_{\text {cells }}\left|\kappa_{\nu}^{\prime}\right|}{N_{\text {cells }}}
$$

Both these quantities are constant for each frequency considered, and provide a measure of the approximate magnitude of the emission and absorption at that frequency. Figure 3 is an example of the variation of these average coefficients and demonstrates the wide variation in radiation properties for different radiative frequencies in a single flowfield. To use these normalizing quantities, Eq. 14 is divided by $\bar{j}_{\nu}$ on both sides, and terms containing $G_{\nu}$ are multiplied by 1 in the form of $\bar{\kappa}_{\nu} / \bar{\kappa}_{\nu}$. A new variable, ${ }_{\nu}=G_{\nu} /\left(\bar{j}_{\nu} \bar{\kappa}_{\nu}\right)$ is defined. Eq. 14 then becomes:

$$
\begin{array}{r}
{\left[\left(\frac{\bar{\kappa}_{\nu}}{\kappa_{\nu}^{\prime}} \vec{\nabla},{ }_{\nu}\right)_{i+1} \cdot \vec{a}_{i+1}-\left(\frac{\bar{\kappa}_{\nu}}{\kappa_{\nu}^{\prime}} \vec{\nabla},{ }_{\nu}\right)_{i} \cdot \vec{a}_{i}\right]_{K}} \\
+\left[\left(\frac{\bar{\kappa}_{\nu}}{\kappa_{\nu}^{\prime}} \vec{\nabla},{ }_{\nu}\right)_{k+1} \cdot \vec{a}_{k+1}-\left(\frac{\bar{\kappa}_{\nu}}{\kappa_{\nu}^{\prime}} \vec{\nabla},{ }_{\nu}\right)_{k} \cdot \vec{a}_{k}\right]_{I} \\
=V\left(3 \bar{\kappa}_{\nu} \kappa_{\nu}^{\prime}, \nu-12 \pi \frac{j_{\nu}^{e}}{\bar{j}_{\nu}}\right)_{I, K}
\end{array}
$$

This formulation reduces the variation of the unknown and coefficients of the equation relative to Eq. 14, making the numerical solution easier. To further normalize the coefficients, this equation is multiplied on both sides by $\kappa_{\nu_{(I, K)}^{\prime}}^{\prime}$.

The flowfield grid is not orthogonal, so the expression of the gradient in finite differences requires a transformation to an orthogonal computational domain as shown in Fig. 4. Transforming to the new $(\xi, \zeta)$ domain, and expanding the gradient of,$\nu$ in second order central differences allows Eq. 17 to be expressed as:

$$
\begin{array}{r}
d_{1}, \nu_{I+1, K+1}+d_{2}, \nu_{I+1, K}+d_{3}, \nu_{I+1, K-1} \\
+d_{4}, \nu_{I, K+1}+d_{5}, \nu_{I, K}+d_{6}, \nu_{I, K-1} \\
+d_{7}, \nu_{I-1, K+1}+d_{8}, \nu_{I-1, K}+d_{9}, \nu_{I-1, K-1} \\
=-12 \pi V_{I, K} \frac{j_{\nu_{I, K}}^{e}}{\bar{j}_{\nu}}
\end{array}
$$

where the coefficients $d_{i}$ are combinations of the geometry and the radiative properties resulting from collecting terms after Eq. 17 is differenced. 
Equation 18 is a matrix equation for the unknown, $\nu_{I, K}$ of the general form $A \bar{x}=\bar{b}$. The numerical solution of this type of equation can be obtained by a number of standard methods. The line Gauss-Seidel algorithm has been selected and applied along each normal grid line to capture the dominant gradients in the radiative properties. This algorithm results in a tridiagonal matrix equation to be solved for each normal grid line. The solution of such an equation can be obtained using the Thomas algorithm ${ }^{15}$ with specification of appropriate boundary conditions as discussed below. To ensure convergence for this problem underrelaxation is necessary. It is introduced by defining a corrected value for the update of ,$\nu$ at the $n+1$ time level as

$$
,{ }_{\nu_{I, K}}^{n+1^{\prime}}={ }_{\nu_{I, K}}^{n}+r\left(,{ }_{\nu_{I, K}}^{n+1}-,{ }_{\nu_{I, K}}^{n}\right)=(1-r),{ }_{\nu_{I, K}}^{n}+r,{ }_{\nu_{I, K}}^{n+1}
$$

where $r$ is the relaxation parameter and is less than one for underrelaxation. The selection of $r$ is discussed below. , ${ }_{\nu_{I, K}}^{n+1}$ is substituted from Eq. 18 to obtain a new form of the matrix equation for,${ }_{\nu_{I, K}}^{n+1^{\prime}}$. Additional details of the derivation and the form of the coefficients $d_{i}$ are given in Ref. 16.

So far this development has ignored the singularity introduced in Eq. 12 with the division by $\kappa_{\nu}^{\prime}$. Two problems arise in this connection. The first is a loss in diagonal dominance in Eq. 18 resulting from the change in sign of $\kappa_{\nu}^{\prime}$ across a cell face. The second is the singularity itself.

To obtain convergence at these frequencies, a time variation term, $C \partial G_{\nu} / \partial t$, is introduced on the left-hand-side of Eq. 12. At the converged steady state this term is zero, but during the iterative solution it adds an additional term to the diagonal coefficient: $d_{5} \Rightarrow d_{5}+C \bar{\kappa}_{\nu}$. The term $C \bar{\kappa}_{\nu},{ }_{\nu_{I, K}}$ also appears on the right-hand-side. The variable $C$, which is related to a time step, can then be chosen such that diagonal dominance is recovered.

To avoid the singularity in Eq. 12, note from the original equation, Eq. 9, that when $\kappa_{\nu}^{\prime}=0, \nabla G_{\nu}=0$. To enforce this in the numerical solution, $\kappa_{\nu}^{\prime}=0$ is assumed to occur $a t$ the cell face. The appropriate terms from Eq. 17 are then dropped for cell faces where $\kappa_{\nu}^{\prime}$ crosses zero. 
This approach allows solutions to be obtained for all frequencies with a single algorithm: $C$ is set to zero when $\kappa_{\nu}^{\prime}$ is everywhere positive, and to a value ensuring diagonal dominance when it is not; and the appropriate terms in the coefficients $d_{i}$ are set to zero where $\kappa_{\nu}^{\prime}$ crosses zero. The choice of the value of $C$ is discussed below.

\section{Boundary Conditions}

Boundary conditions in the 3-D medium are illustrated in Fig. 2. The treatment of each is discussed below.

Reflection boundary conditions should apply at the symmetry boundary. This boundary is a singularity in the computational grid, however, where one surface of a grid cell has zero area. Referring back to Eq. 17, this means the second term on the first line disappears. The remaining $\xi$-derivative terms at the $I$ grid line are replaced by first order forward differences to avoid differencing across the singularity. ${ }^{17}$ The appropriate form of the coefficients $d_{i}$ is given in Ref. 16.

For the medium only intensity, the wall boundary behaves as a cold wall. The Marshak cold wall boundary condition for the P-1 method is:

$$
2\left[\frac{2}{\varepsilon_{\nu}}-1\right] \vec{q}_{\nu} \cdot \hat{n}+G_{\nu}=0
$$

Replacing $\vec{q}_{\nu}$ in this equation using Eq. 9 leads to a differential equation in the single unknown $G_{\nu}$, which can be transformed to an equation for,$\nu$. This equation can be differenced at the wall boundary by recognizing that the grid lines are normal to the body surface. The $\vec{\nabla}, \nu \cdot \hat{n}$ term is then simply the gradient of,$\nu$ along the grid lines. This difference is best expressed in physical coordinates with a first order forward scheme, and can be incorporated in the Gauss-Seidel solution scheme with underrelaxation. The details are given in Ref. 16.

Along the outflow "boundary", the value of , $\nu$ must be approximated. This is an arbitrary computational boundary through which radiating gas and radiation (inwardly and outwardly directed) both pass. The flowfield boundary condition used in LAURA is a zeroth order extrapolation (i.e., the derivatives of the flow variables are assumed to be zero across 
this boundary). This boundary condition is known to provide better stability for CFD computations than other possible formulations, and so might be adopted for the radiation method. Several alternatives are possible at this boundary, such as a constant slope of ${ }_{\nu}(I, K)$ or constant radiation intensity. These various boundary conditions are found to have only a local effect on the solution. The selection of the "correct" boundary condition is hampered by grid distortion and a lack of resolution in the shoulder area of currently available CFD solutions. The choice is therefore made on the basis of stability rather than accuracy. The boundary condition that is applied in this work is

$$
G_{\nu}(\text { outflow boundary })=0
$$

Though incorrect, this condition enhances stability while providing the correct trend of a fall-off in $G_{\nu}$ around the shoulder. Future work with complete flowfields will move the outflow boundary downstream in the wake, where its influence on the flowfield will be much smaller.

The freestream "boundary" is transparent and only outgoing radiation is considered. The freestream is assumed to be non-emitting. With these assumptions, this boundary can be modeled as a cold wall with complete absorption $\left(\varepsilon_{\nu}=1.0 \mathrm{in}\right.$ Eq. 20$)$. Differencing using a central difference for the $\xi$-direction and a first-order backward difference for the $\zeta$-direction allows this equation to be written in the line Gauss-Seidel form. Underrelaxation can then be added as well. The final expression is reported in Ref. 16.

For the freestream boundary at the axis of symmetry, a reflection boundary condition for $G_{\nu_{I-1, F}}$ is used. Using a first order forward difference at this point was found to lead to nonphysical results.

\section{Radiative Heat Flux to Wall}

The radiative flux to the wall due to emission in the medium can be found from solving Eq. 9 for $\vec{q}_{\nu}$. The value of $G_{\nu}$ is obtained from the solution of the finite volume problem, , $\nu$, multiplied by the normalizing factor $\left(\bar{j}_{\nu} \bar{\kappa}_{\nu}\right)$. To obtain the flux directed to the wall, the dot product of $\vec{q}_{\nu}$ with the wall-directed surface normal is required. Again recalling that the grid 
lines are normal to the body surface, the gradient of $G_{\nu}$ can be expressed with a one-sided difference along the normal grid lines. As above, this is best done in physical coordinates:

$$
q_{\nu_{w}}=-\frac{1}{3 \kappa_{\nu_{I, w}}^{\prime}}\left(\frac{d G_{\nu}}{d n}\right)_{w}
$$

The total heat flux to the wall at each grid location is then obtained by a numerical integration of Eq. 22 over the spectral range considered.

\section{Convergence Criterion}

The numerical solution of this set of difference equations requires the selection of an appropriate criterion to test for convergence. Because the radiative properties vary over many orders of magnitude at a single frequency, the usual error criteria must be modified. A local error function is defined as:

$$
L_{I, K}=\frac{\left|R^{n}\right|}{\operatorname{mean}\left(,{ }_{\nu}^{n}\right)}
$$

where $R^{n}$ is the residual of the difference equation, Eq. 18. The mean of,${ }_{\nu}^{n}$ is the average of the minimum and maximum values of,${ }_{\nu}^{n}$ for all $I$ and $K$ on the radiation grid. (For frequencies where $\kappa_{\nu}^{\prime}$ changes sign, it is defined as half the maximum value of ${ }_{\nu_{I, K}}^{n}$.) This definition reduces the error in regions where, ${ }_{\nu_{I, K}}^{n}$ is small, relative to the error that would be computed from the residual alone. Though the solution may not be completely converged at locations where, ${ }_{\nu_{I, K}}^{n}$ is small, these locations contribute little to the coupling with the flowfield. Therefore, the lack of complete convergence at these locations is deemed acceptable to reduce the computation time.

Each frequency is converged individually, since the rate of convergence depends on the magnitude of the optical depth and varies widely for different frequencies. A typical convergence history for a single frequency is shown in Fig. 5. For other frequencies convergence requires 50 to several hundred iterations, and for a few frequencies even thousands of iterations. 


\section{Selection of Solution Parameters: $r, C$}

The relaxation parameter required to obtain a stable solution varies from frequency to frequency. No simple dependence of $r$ on any radiative property has so far been discovered. The value $r=0.5$ has therefore been selected as a compromise, since it is found to provide generally good stability and convergence rates for all frequencies.

The constant $C$ in the time term added for frequencies with $\kappa_{\nu}^{\prime}$ crossing zero must be selected to recover diagonal dominance. Since $C$ is related to the inverse of the time step, it must be kept as small as possible or the solution does not progress. To minimize the additional logic involved, an initial guess is made which will set $d_{5}=1.1 d_{5}$. $C$ is then successively doubled if tests indicate lack of diagonal dominance.

It should be noted that the objective of this study is to demonstrate the feasibility of the method; not to optimize the numerical solution. Further improvements to this logic are almost surely possible and will be pursued as the method is used.

\section{Results and Discussion}

The LORAN radiation method ${ }^{1}$ is used to obtain all the radiation predictions shown in this paper. Infrared, visible, and ultraviolet radiation from 0.31 to $16.5 \mathrm{eV}$ is computed on an optimized spectrum of 1066 points. ${ }^{1}$ LAURA flowfield solutions provide the nonequilibrium gas conditions. Coupling effects are not included in this study.

\section{Mars Return}

A flowfield solution has been obtained for one of a number of possible flight conditions identified for the return from a mission to Mars. ${ }^{18}$ It consists of a $60^{\circ}$ sphere cone with a $1.08 \mathrm{~m}$ nose radius flying at $80 \mathrm{~km}$ altitude with a velocity of $12 \mathrm{~km} / \mathrm{sec}$. Results have been obtained for this case using both tangent slab transport and the MDA method.

Figure 6 compares the wall radiation flux predictions from the two transport methods. The MDA result is found to be consistently lower than the tangent slab result. This results from a combination of geometric effects and flowfield property gradients. Figure 7 presents 
the ratio of the MDA to the tangent slab solution. It shows that the tangent slab solution applies best at the stagnation point (within 6 percent of MDA), and becomes progressively worse along the flank. The odd behavior at the shoulder is a result of grid problems in the flowfield solution used to obtain these results (these problems have since been fixed in LAURA). The qualitative trends of the two methods are similar, showing the radiative flux increasing with distance from the sphere-cone juncture. This increase is unlike the behavior of convective heating on such a body and results because radiation is a volumetric quantity. As suggested in Fig. 4, the standoff distance is much larger on the flank than at the stagnation point of this vehicle, so the volume of radiating gas is greatly increased. The vibrational temperature in this nonequilibrium flowfield is still high in this region, so the radiative emission is strong.

The other variable of interest for coupled flowfields is the divergence of the radiative flux in the shock layer. To compare the two transport methods, Figs. 8 and 9 show contour plots of $\nabla \cdot \vec{q}_{\mathrm{R}}$ in the computational domain (see Fig. 4), since it is difficult to see details in the physical domain. For a viscous flow, this has the effect of stretching the boundary layer region, which extends to $\zeta / \zeta_{\max }$ of 0.3 to 0.4 in these plots. The position of the first 0.0 contour level in the boundary layer region is affected by different gridding in the two cases, and is not indicative of a difference in the solutions. The tangent slab result shown in Fig. 8 is in fact just the derivative of $q_{\mathrm{R}}$ along each normal grid line, so the values at neighboring grid lines do not influence each other. The localized hot spot near $\xi / \xi_{\max }=0.8$ may be an artifact of this 1-D assumption. The grid problems in the shoulder area are evident from the distorted contours near $\xi / \xi_{\max }=1$, and in fact the last grid line is not included in the MDA solution. In all other aspects, the solutions from the two transport methods are qualitatively and often even quantitatively similar, giving confidence in the MDA method.

The tangent slab calculation for this case required 7 CPU minutes on an IRIS 4D/340 workstation. The MDA result took 35.8 CPU hours, or about 300 times longer. While this is a large penalty for the slight difference obtained in the results, several comments can be 
made. Since the IRIS workstation has four processors, the MDA result required only about 10 hours of real elapsed time. The speedup factor that could be obtained by running the method on the Cray is expected to be especially large, because it will allow the use of single rather than double precision arithmetic. Finally, the MDA algorithm is in its early stages, and significant reductions in computing time will probably be obtained as the algorithm matures. In particular, this result was obtained using a larger radiation subgrid (32 grid points normal to the body) than was suggested in Ref. 1 in order to obtain the best results for the evaluation of the method. Taking these factors into account, the MDA method has potential for providing accurate heating distributions in important cases, though it may never be used as commonly as the tangent slab method. More important, though, it can be used in flow regions (such as wakes) where the tangent slab method cannot be applied.

\section{Geosynchronous Return}

Results were also obtained at a flight condition representative of return from a geosynchronous orbit. The aerobrake configuration is a raked cone with a blunted elliptic nose (AFE). ${ }^{19}$ Several studies of nonequilibrium radiative heating for this configuration have been made. ${ }^{20-23}$ An axisymmetric LAURA flowfield solution in which this geometry was modeled by a sphere with an equivalent nose radius of $2.16 \mathrm{~m}^{24}$ was generated for this study. ${ }^{25}$ Results will only be given for the stagnation region, since the sphere quickly deviates from the AFE geometry. The flowfield models used to obtain this solution are the baseline models of Ref. 26, including the use of the Park-87 chemical kinetics. The wall temperature was assumed to be constant at $1750 \mathrm{~K}$.

Radiation predictions were obtained for this case, again using both tangent slab and MDA transport methods. The tangent slab calculation, including calculation of the radiation properties $\kappa_{\nu}^{\prime}$ and $j_{\nu}^{e}$, was completed in about 45 minutes of actual elapsed time on a Silicon Graphics 4D/320, while the MDA result required about 4 hours of elapsed time. Including the calculation of the radiation properties in the comparison means that the MDA method 
requires just over 5 times more $\mathrm{CPU}$ time than the tangent slab method for this case. It is anticipated that the MDA run time can be further reduced as the code matures, as discussed above.

The radiative heating rates predicted for this configuration at $9.3 \mathrm{~km} / \mathrm{s}$ and $76 \mathrm{~km}$ altitude are shown in Fig. 10. These predictions are in the same range as earlier computations ${ }^{9,27}$ (note that different nose radii have been used for these computations). The MDA prediction is again lower than the tangent slab result, with the dip at the outflow boundary to be attributed to the outflow boundary condition used, rather than any physical effect.

The radiative flux divergence for this flowfield is presented in Figs. 11 and 12 for the tangent slab and MDA transport, respectively. This time the physical domain is used, since details in the nose region are apparent in these coordinates. The approximations inherent in the tangent slab solution can be observed in this case as significant differences in the profiles along adjacent surface normal lines. As mentioned earlier, the tangent slab method employs a numerical differentiation to obtain $d q_{\mathrm{R}} / d n$ while the MDA method requires no such differentiation. This may add to the error already incurred by ignoring the variation of the radiation properties in the direction parallel to the surface. This result suggests that for some flowfield cases, the MDA result might provide a smoother variation of the radiative flux divergence when flowfield coupling is included, and thus might enhance the stability of coupled solutions.

The amount of heating to geosynchronous return vehicles resulting from radiation in the ultraviolet (UV) portion of the spectrum has been the subject of some debate. The distribution of radiation predicted by the LORAN method is shown in Fig. 13, using both the tangent slab and MDA transport models. The relative importance of different spectral regions varies only slightly between the two transport methods. Both curves indicate that just over half of the radiative heating experienced by this vehicle results from the UV portion of the spectrum. This is a significant fraction of the total. Radiation in the UV spectral range is highly self-absorbed. If the amount of self-absorption is mispredicted only slightly, 
the wall radiative flux may be significantly increased.

\section{Conclusions}

A Modified Differential Approximation (MDA) method for computing 3-D radiative transport in axisymmetric thermochemical nonequilibrium flows is developed. It employs the P-1 approximation to reduce the integro-differential governing equation of radiative transport to a set of partial differential equations. The numerical solution of these equations in a finite volume algorithm with real radiative property variations is discussed. The method is assessed in forebody flowfields by comparison to the commonly used tangent slab transport approximation. The MDA method is intended for eventual use in flow areas, such as wakes, where the tangent slab approximation is known to be invalid.

Predictions of the radiative heat flux to the forebody wall of an axisymmetric vehicle are obtained from the two transport methods for two representative flowfields. Comparison of the results shows qualitative agreement, with wall radiative heating predictions from the MDA method about 10 to 20 percent lower than from the tangent slab. The differences increase away from the stagnation region due to geometric and flowfield gradient effects not accounted for in the tangent slab approximation. The variation of the divergence of the radiative heat flux, which appears in the energy equation to couple the radiation and flowfield properties, is also examined and found in some cases to be smoother in the MDA method. In addition to providing more accurate coupled results, this feature of the MDA method has the potential to enhance the stability of coupled solutions.

\section{Acknowledgments}

Dr. Peter Gnoffo, Dr. Robert Mitcheltree, and William Kleb, all of the Aerothermodynamics Branch at NASA Langley Research Center, provided invaluable help to this effort by patient discussions of the numerical solution of the MDA equation. 
Dr. H. A. Hassan was supported in part by NASA's Cooperative Agreement NCCI-112, the Hypersonic Aerodynamic Program Grant NAGW-1022 funded by NASA, and the Mars Mission Research Center funded by NASA's Grant NAGW-1331.

\section{References}

${ }^{1}$ Hartung, L. C., "Development of a Nonequilibrium Radiative Heating Prediction Method for Coupled Flowfield Solutions," AIAA Paper 91-1406, Honolulu, HI, June, 1991.

${ }^{2}$ Nicolet, W. E., "Rapid Methods for Calculating Radiation Transport in the Entry Environment," NASA CR 2528, April, 1975.

${ }^{3}$ Arnold, J. O., Cooper, D. M., Park, Chul, and Prakash, S. G., "Line-by-Line Transport Calculations for Jupiter Entry Probes," Progress in Astronautics and Aeronautics, Vol. 69, AIAA, 1980, pp. 52-82.

${ }^{4}$ Balakrishnan, A., and Nicolet, W. E., "Galileo Probe Forebody Thermal Protection: Benchmark Heating Environment Calculations," AIAA Paper 81-1072, Palo Alto, CA, June, 1981.

${ }^{5}$ Bolz, C. W. ,Jr., “A Marching Method for Calculating Line and Continuum Radiation in High Energy Flowfields," NASA CR 3046, June, 1979.

${ }^{6}$ Carlson, L. A., "Approximation for Hypervelocity Nonequilibrium Radiating, Reacting, and Conducting Stagnation Regions," AIAA Paper 88-2672, San Antonio, TX, June, 1988.

${ }^{7}$ Gupta, R. N., Lee, K. P., Moss, J. N., and Sutton, K., "A Viscous-Shock-Layer Analysis of the Martian Aerothermal Environment," AIAA Paper 91-1345, Honolulu, HI, June, 1991.

${ }^{8}$ Olstad, W. B., "Nongray Radiating Flow about Smooth Symmetric Bodies," AIAA Journal, Vol. 9, No. 1, January, 1971, pp. 122-130.

${ }^{9}$ Candler, G., and Park, Chul, "The Computation of Radiation from Nonequilibrium Hypersonic Flows," AIAA Paper 88-2678, San Antonio, TX, June, 1988.

${ }^{10}$ Hamilton, H. H., II and Greendyke, R. B., "Calculation of Convective and Radiative Heating on the Forebody Heatshield of the Aeroassist Flight Experiment Vehicle," NASA 
TM 104103, May, 1991.

${ }^{11}$ Fuehrer, P., and Edwards, D., "Computational Methodology for Radiation Heat Transfer in the Flowfield of an AOTV," AIAA Paper 91-1407, Honolulu, HI, June, 1991.

${ }^{12}$ Modest, M. F., "Modified Differential Approximation for Radiative Transfer in General Three-Dimensional Media," Journal of Thermophysics and Heat Transfer, Vol. 3, No. 3, July, 1989, pp. 283-288.

${ }^{13}$ Gnoffo, P. A., Gupta, R. N., and Shinn, J. L., "Conservation Equations and Physical Models for Hypersonic Air Flows in Thermal and Chemical Nonequilibrium," NASA TP 2867, February, 1989.

${ }^{14}$ Gnoffo, P. A., “An Upwind-Biased, Point-Implicit Relaxation Algorithm for Viscous, Compressible Perfect-Gas Flows," NASA TP 2953, February, 1990.

${ }^{15}$ Anderson, D. A., Tannehill, J. C., and Pletcher, R. H., Computational Fluid Mechanics and Heat Transfer, Hemisphere Publishing Corporation, New York, 1984.

${ }^{16}$ Hartung, L. C., "Nonequilibrium Radiative Heating Prediction Method for Aeroassist Flowfields with Coupling to Flowfield Solvers," PhD Thesis, North Carolina State University, 1991.

${ }^{17}$ Gnoffo, P. A., Private Communication, 1991.

${ }^{18}$ Mitcheltree, R. A., and Gnoffo, P. A., "Thermochemical Nonequilibrium Issues for Earth Reentry of Mars Mission Vehicles," AIAA Paper 90-1698, Seattle, WA, June, 1990.

${ }^{19}$ Cheatwood, F. M., DeJarnette, F. R., and Hamilton, H. H., II, “Geometrical Description for a Proposed Aeroassist Flight Experiment Vehicle," NASA TM-87714, July, 1986.

${ }^{20}$ Carlson, L. A., Bobskill, G. J., and Greendyke, R. B., "Comparison of VibrationDissociation Coupling and Radiative Heat Transfer Models for AOTV/AFE Flowfields," AIAA Paper 88-2673, San Antonio, TX, June, 1988.

${ }^{21}$ Moss, J. N., and Price, J. M., "Direct Simulation of AFE Forebody and Wake Flow with Thermal Radiation," NASA TM 100673, September, 1988.

${ }^{22}$ Moss, J. N., Bird, G. A., and Dogra, V. K., "Nonequilibrium Thermal Radiation for 
an AFE Vehicle," AIAA Paper 88-0081, Reno, NV, January, 1988.

${ }^{23}$ Gnoffo, P. A., "Code Calibration Program in Support of the Aeroassist Flight Experiment," Journal of Spacecraft $\&$ Rockets, Vol. 27, No. 2, March-April, 1990, pp. 131-142.

${ }^{24}$ Hamilton, H. H., II, Gupta, R. N., and Jones, J. J., "Flight Stagnation-Point Heating Calculations on Aeroassist Flight Experiment Vehicle," Journal of Spacecraft \&3 Rockets, Vol. 28, No. 1, January-February, 1991.

${ }^{25}$ Greendyke, R. B., Private Communication, 1991.

${ }^{26}$ Hartung, L. C., Mitcheltree, R. A., and Gnoffo, P. A., "Stagnation Point Nonequilibrium Radiative Heating and the Influence of Energy Exchange Models," AIAA Paper 91-0571, Reno, NV, January, 1991.

${ }^{27}$ Greendyke, R. B., and Hartung, L. C., "An Approximate Method for the Calculation of Nonequilibrium Radiative Heat Transfer," Journal of Spacecraft $\&$ Rockets, Vol. 28, No. 1, January-February, 1991. 


\section{Figure Captions}

1. Typical High Speed Aerobrake Flowfield

2. Flow Geometry and Radiation Boundary Conditions

3. Variation of $\bar{\kappa}_{\nu}$ and $\bar{j}_{\nu}$ for a Mars Return Flowfield

4. Physical to Computational Domain Transformation

5. Typical Convergence History for MDA Solution

6. Wall Radiative Flux for Mars Return Case

7. Comparison of MDA and Tangent Slab Result

8. Radiative Flux Divergence in Computational Domain for Mars Return Case with Tangent Slab Transport

9. Radiative Flux Divergence in Computational Domain for Mars Return Case with MDA Transport

10. Wall Radiative Flux for Geosynchronous Return

11. Radiative Flux Divergence for Geosynchronous Return with Tangent Slab Transport

12. Radiative Flux Divergence for Geosynchronous Return with MDA Transport

13. Spectral Distribution of Geosynchronous Return Wall Radiative Flux 


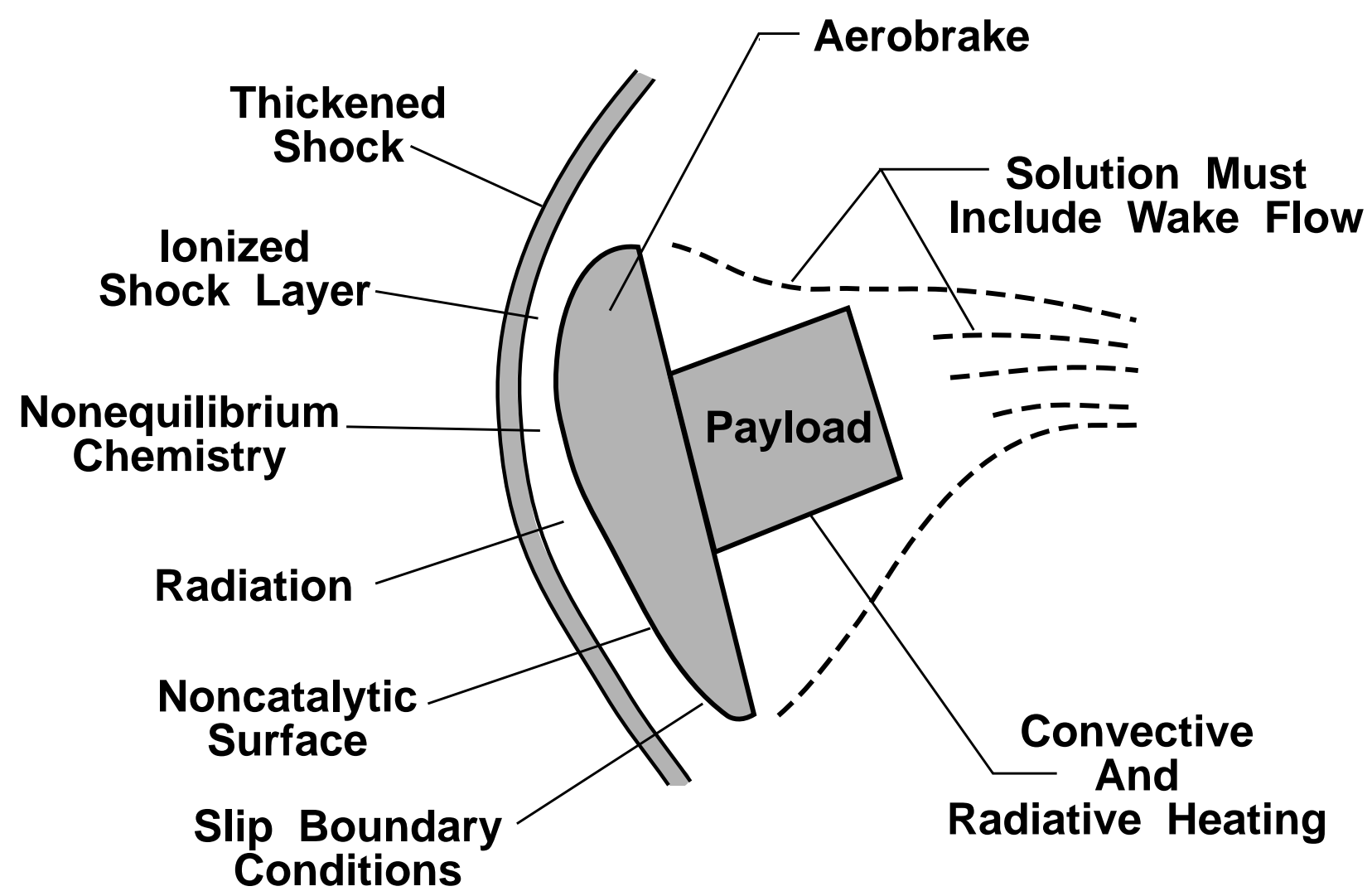

Figure 1: Typical High Speed Aerobrake Flowfield 
Figure 2: Flow Geometry and Radiation Boundary Conditions 


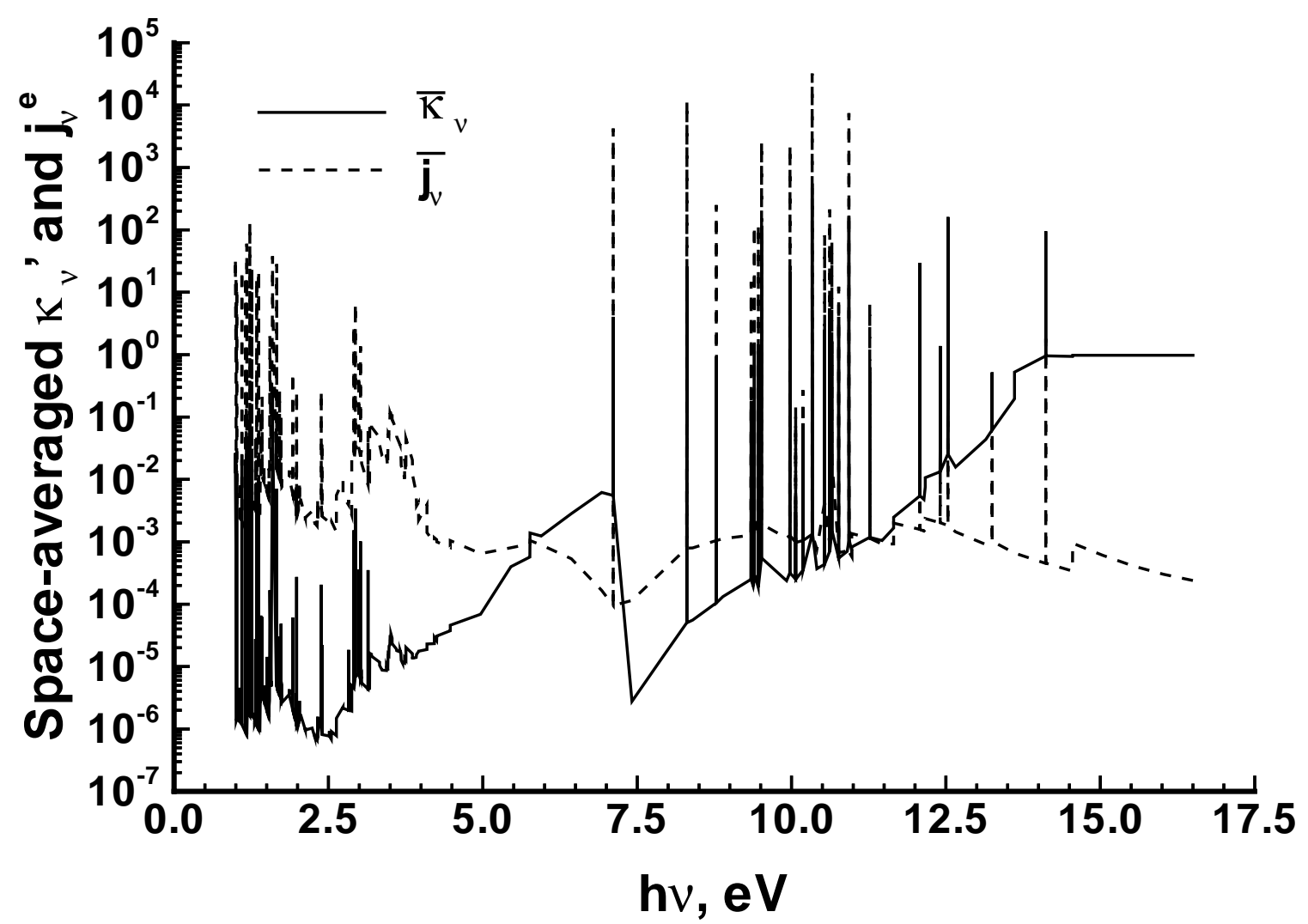

Figure 3: Variation of $\bar{\kappa}_{\nu}$ and $\bar{j}_{\nu}$ for a Mars Return Flowfield 

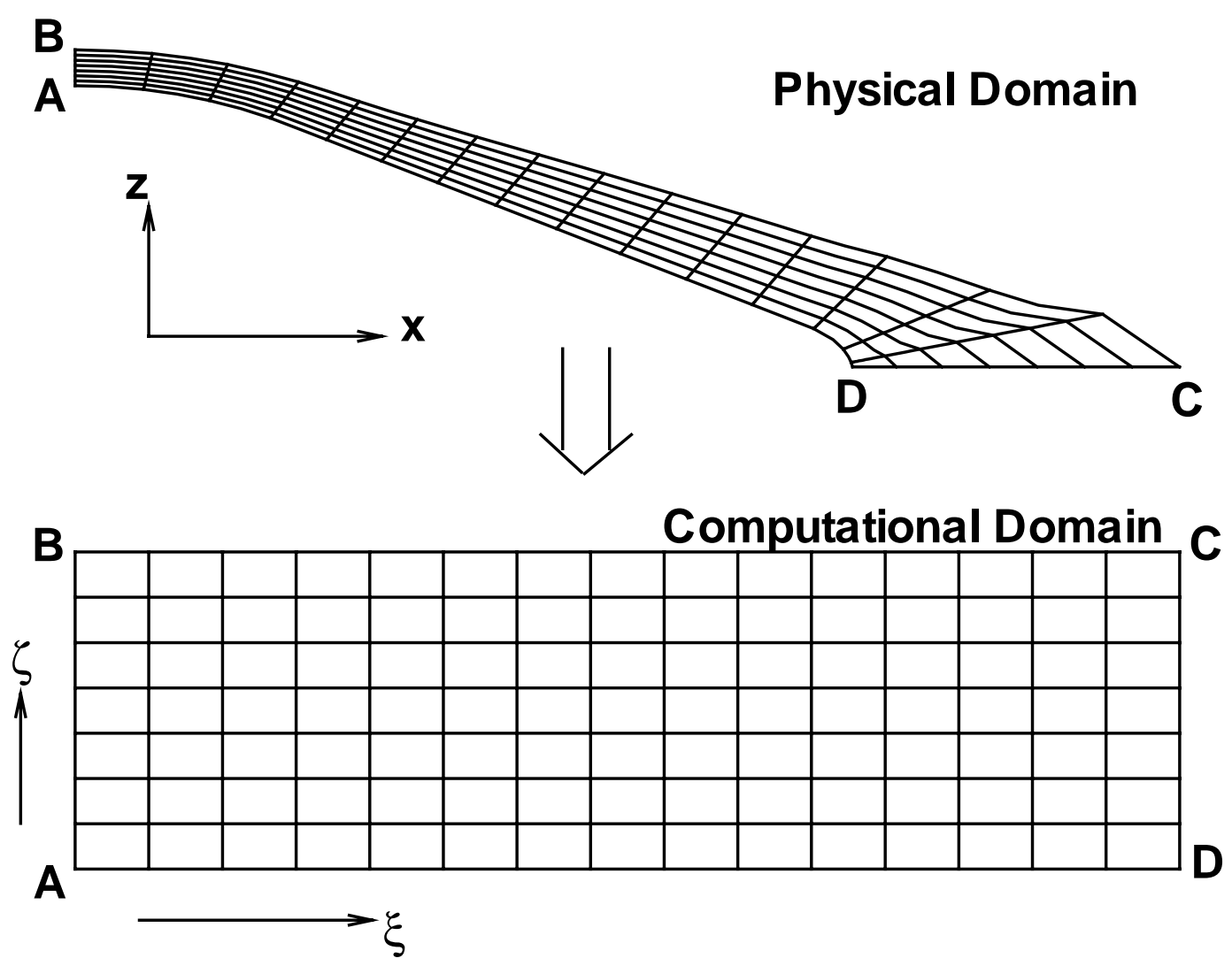

Figure 4: Physical to Computational Domain Transformation 


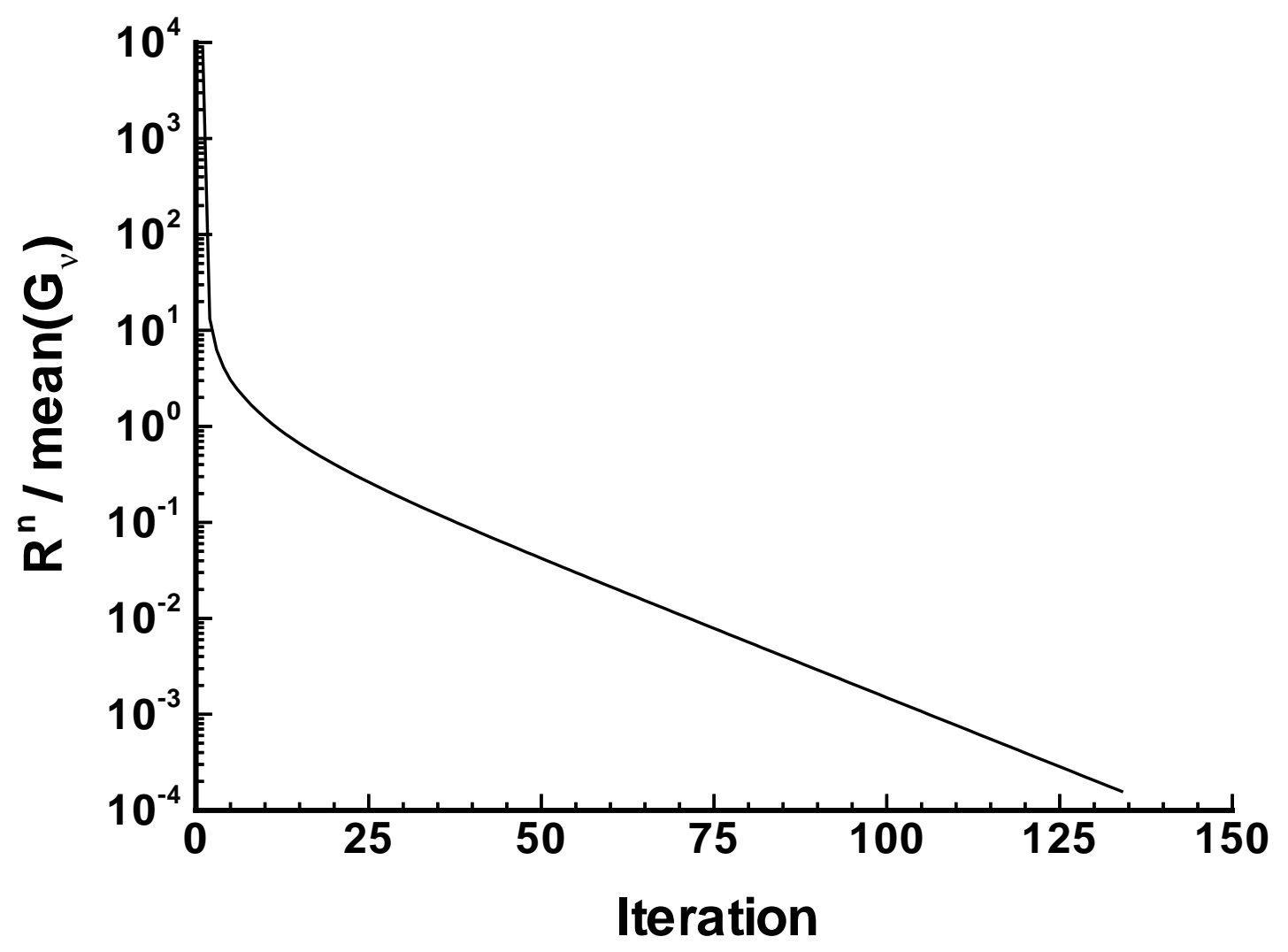

Figure 5: Typical Convergence History for MDA Solution 


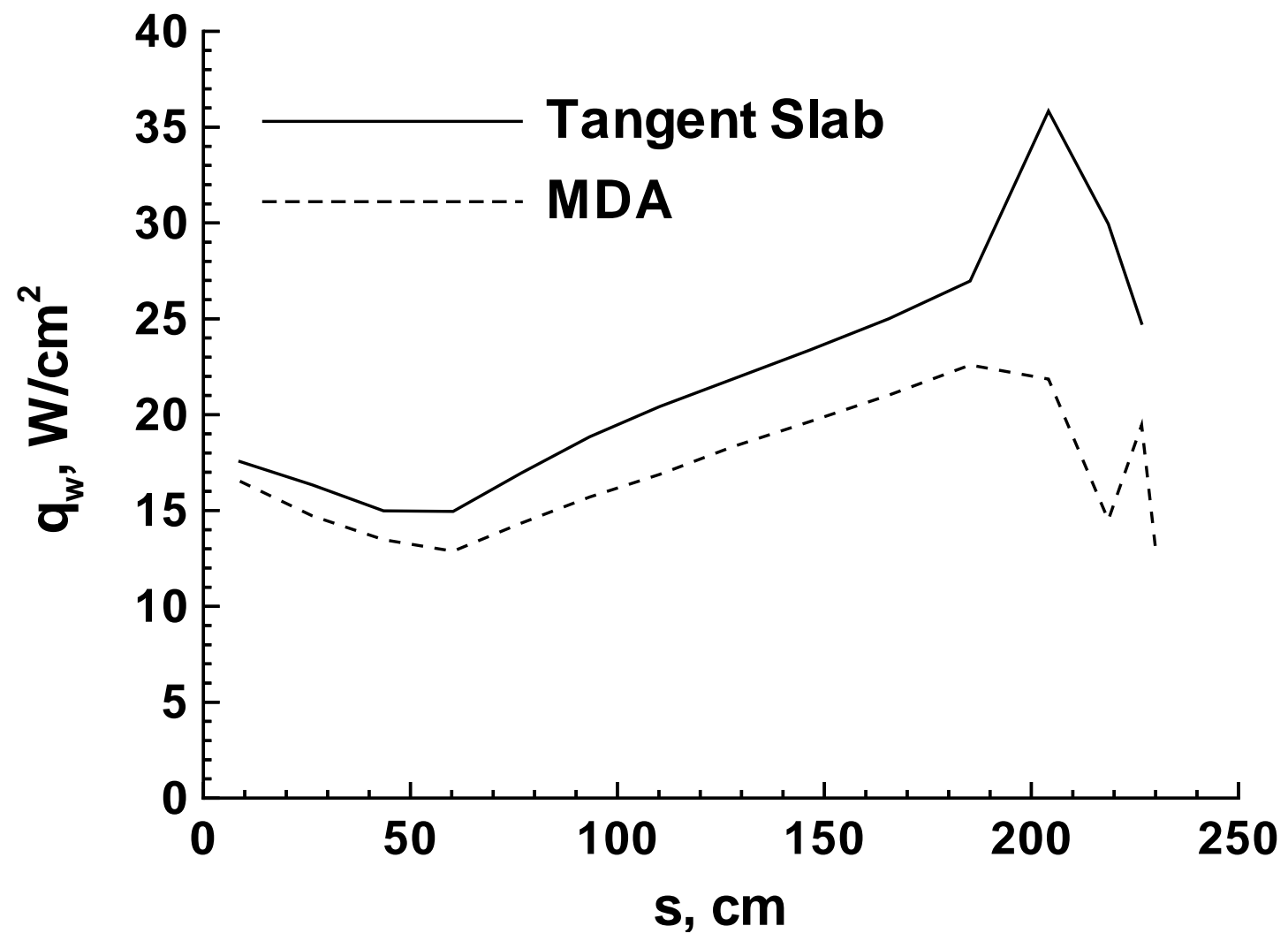

Figure 6: Wall Radiative Flux for Mars Return Case 


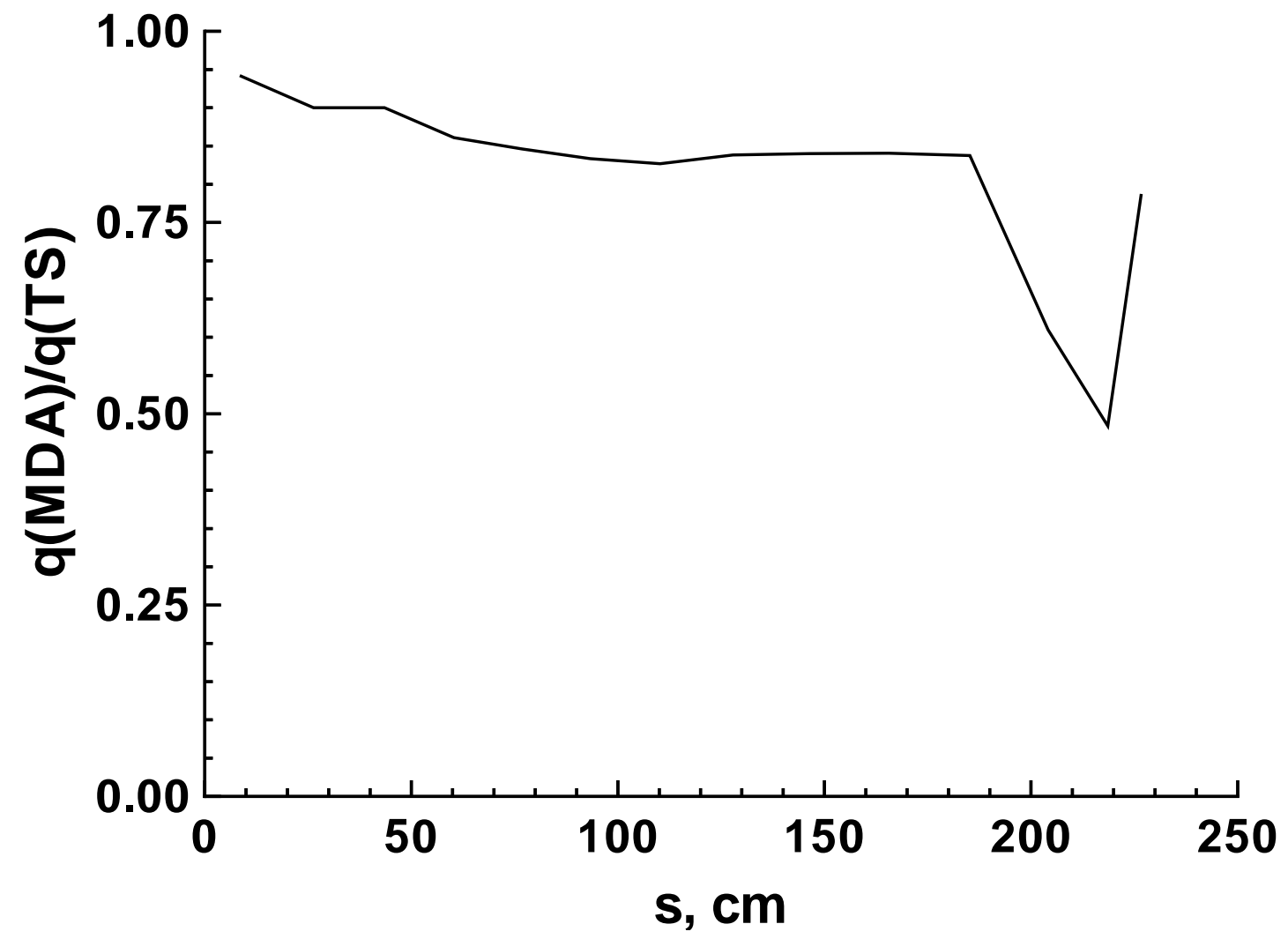

Figure 7: Comparison of MDA and Tangent Slab Result 


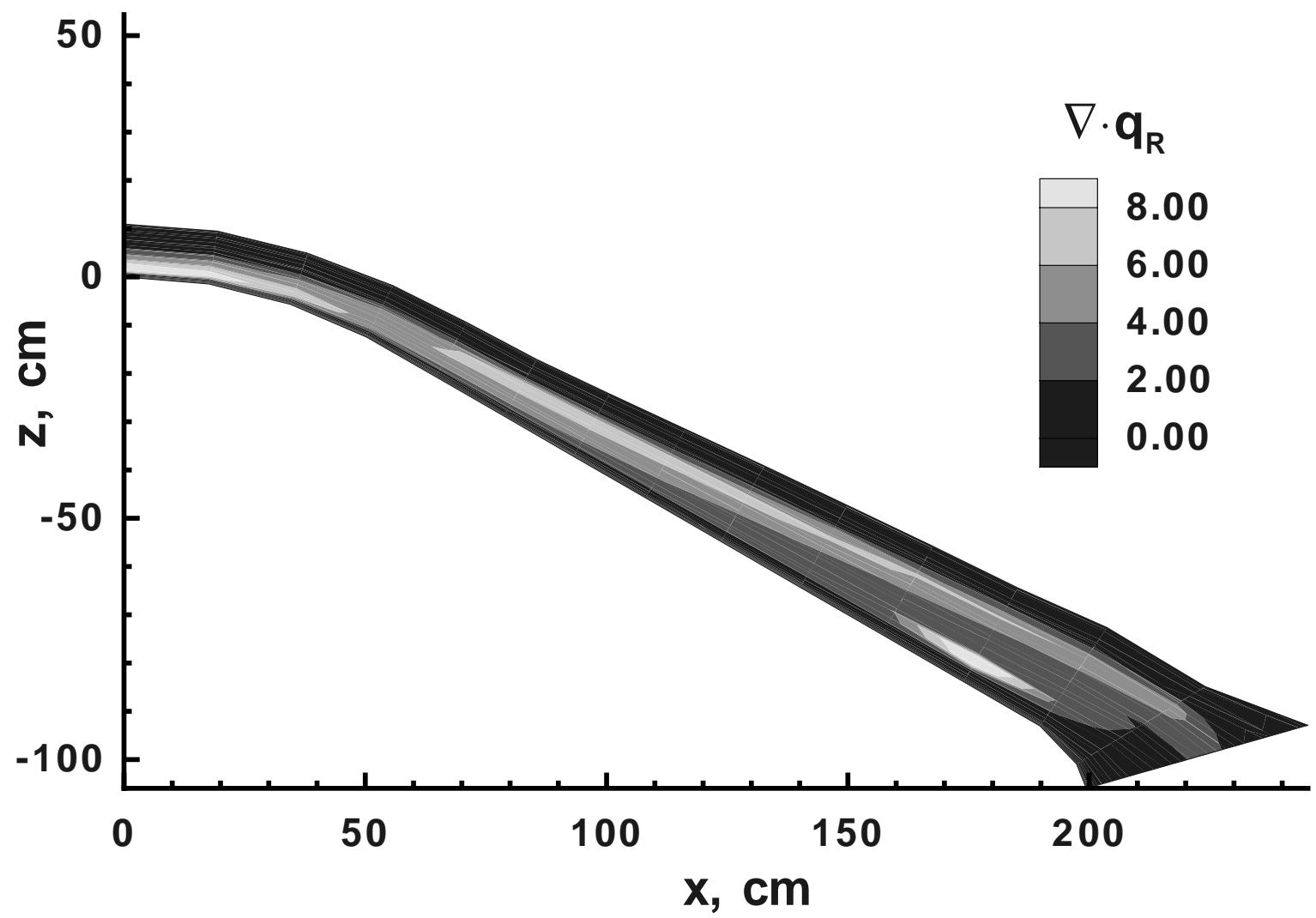

Figure 8: Radiative Flux Divergence in Computational Domain for Mars Return Case with Tangent Slab Transport 


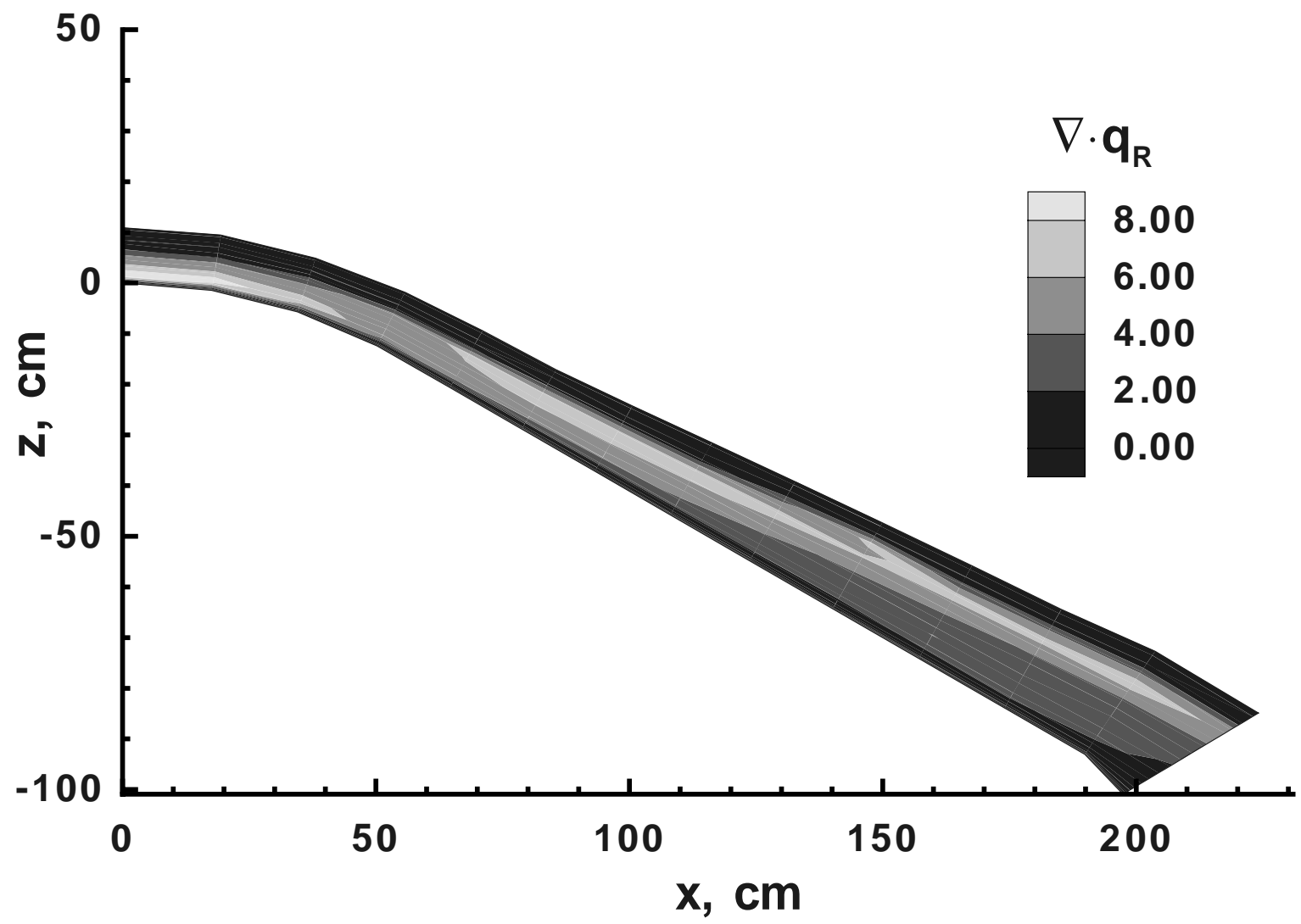

Figure 9: Radiative Flux Divergence in Computational Domain for Mars Return Case with MDA Transport 


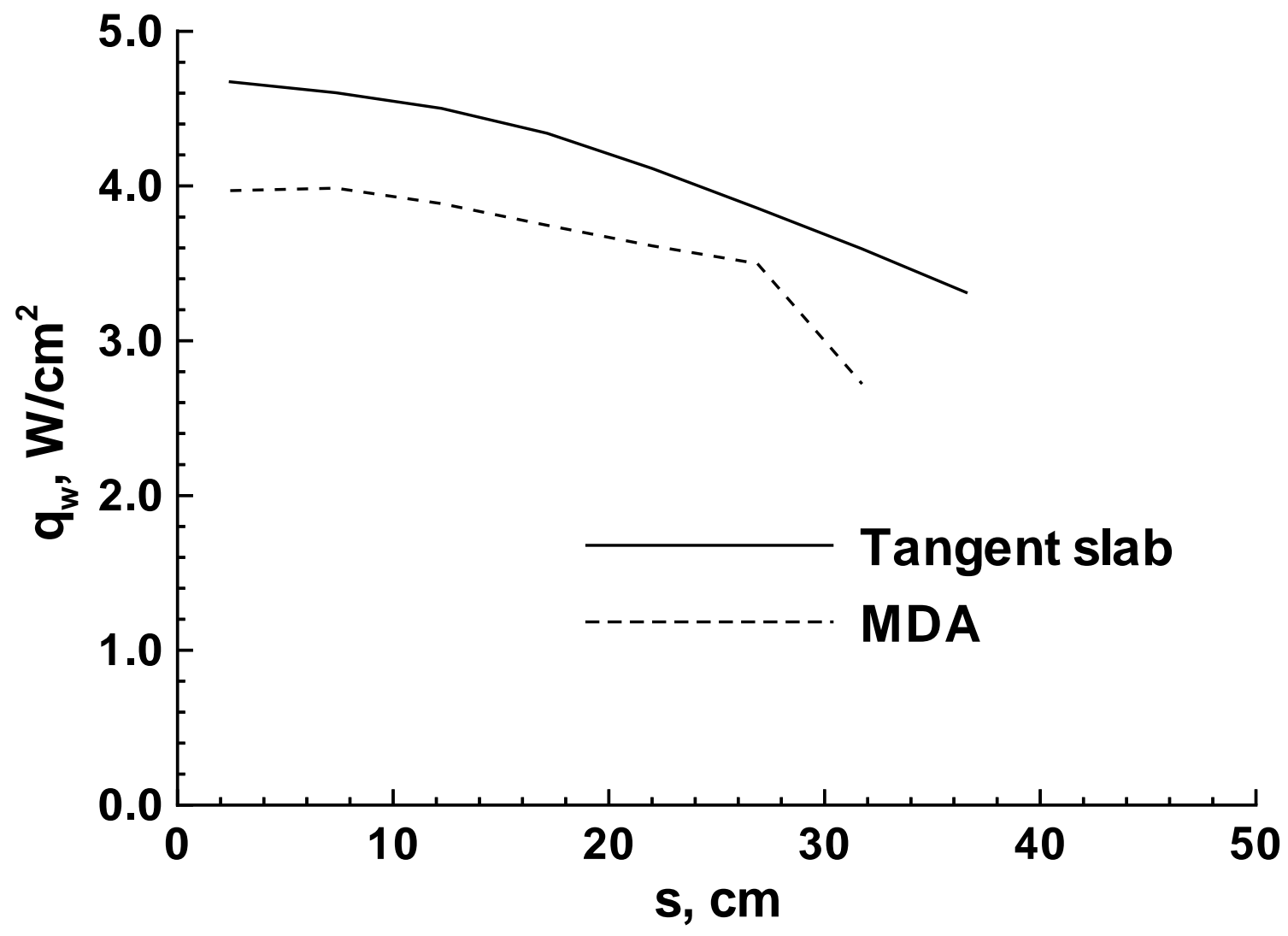

Figure 10: Wall Radiative Flux for Geosynchronous Return 


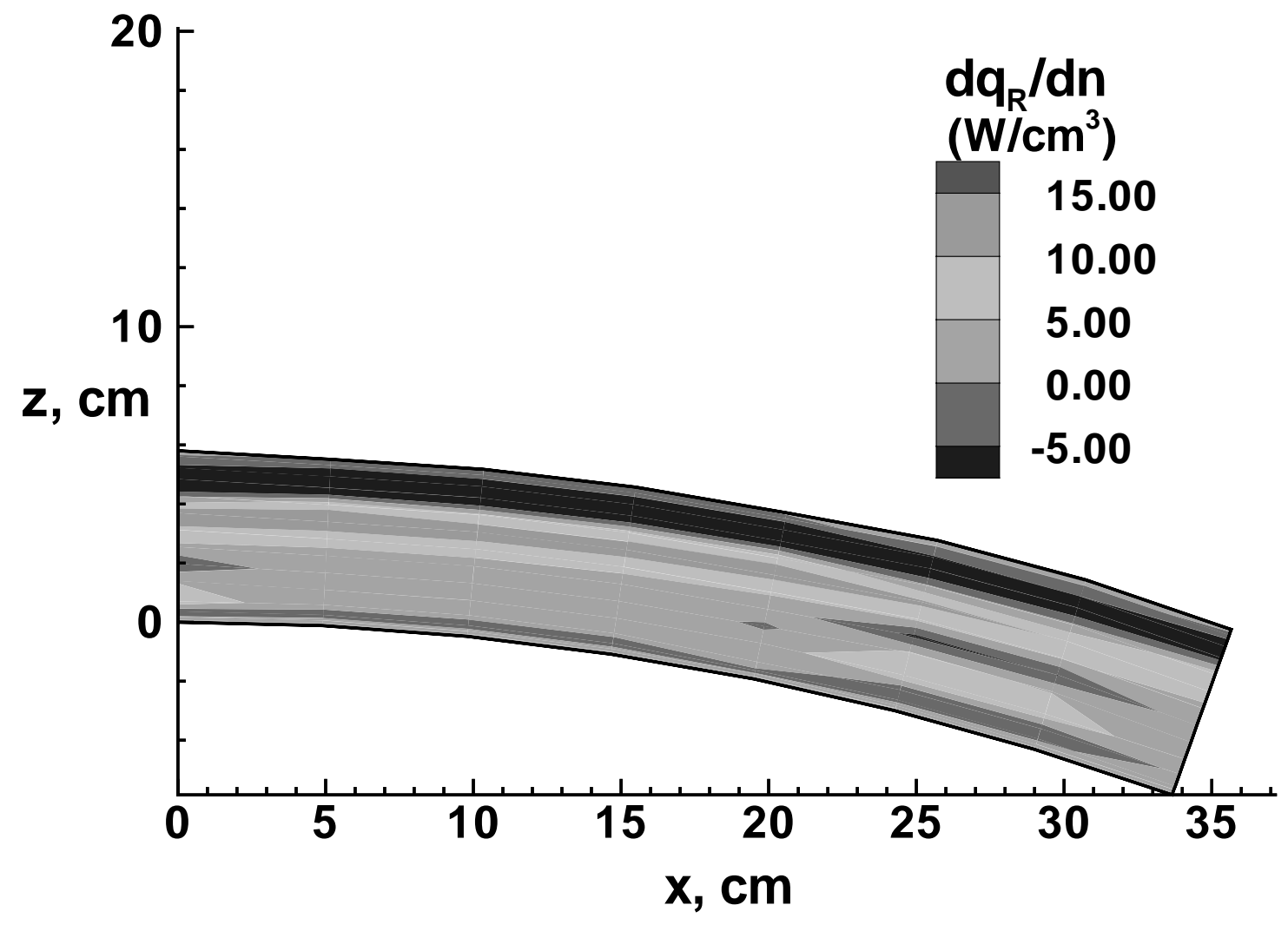

Figure 11: Radiative Flux Divergence for Geosynchronous Return with Tangent Slab Transport 


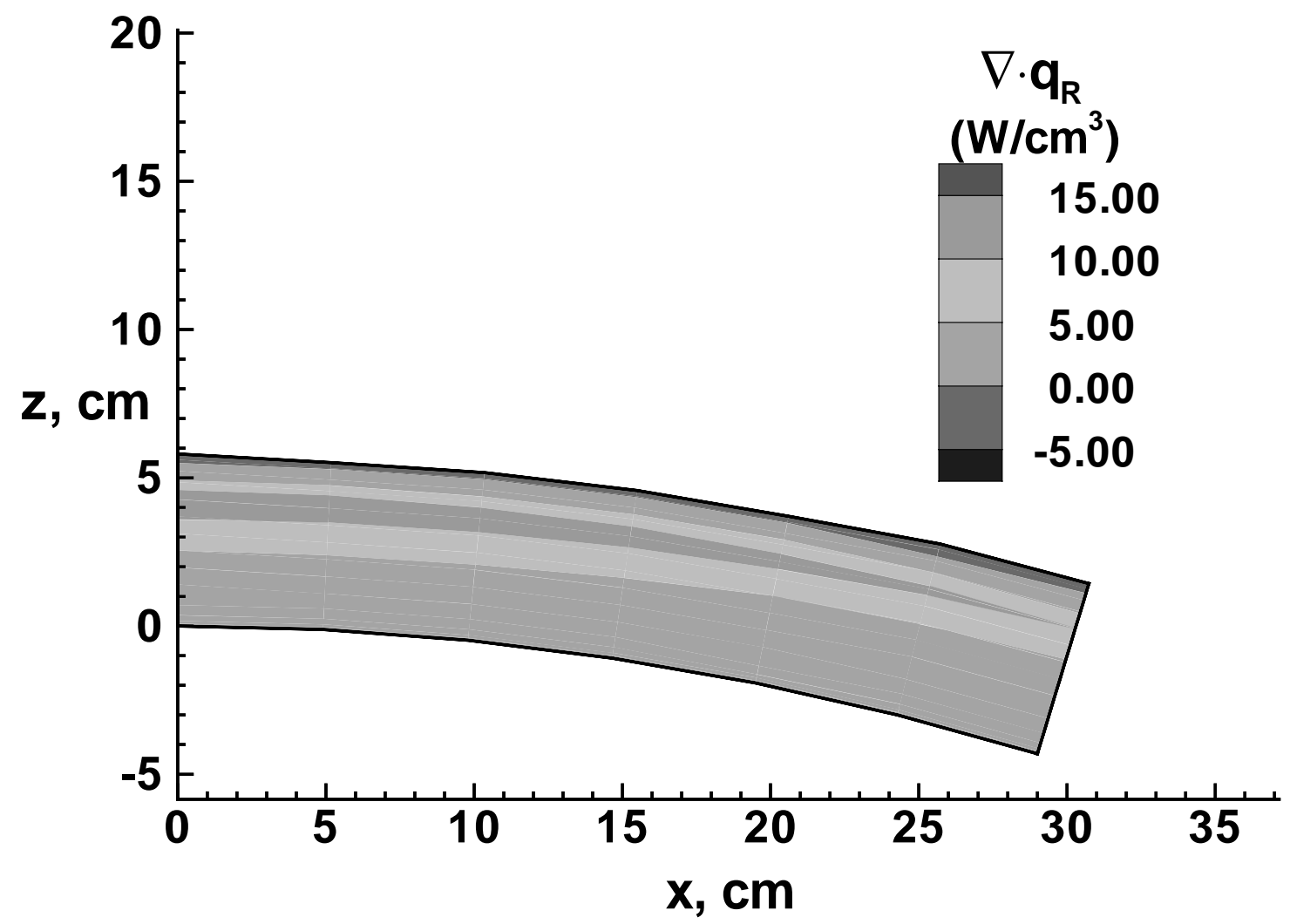

Figure 12: Radiative Flux Divergence for Geosynchronous Return with MDA Transport 


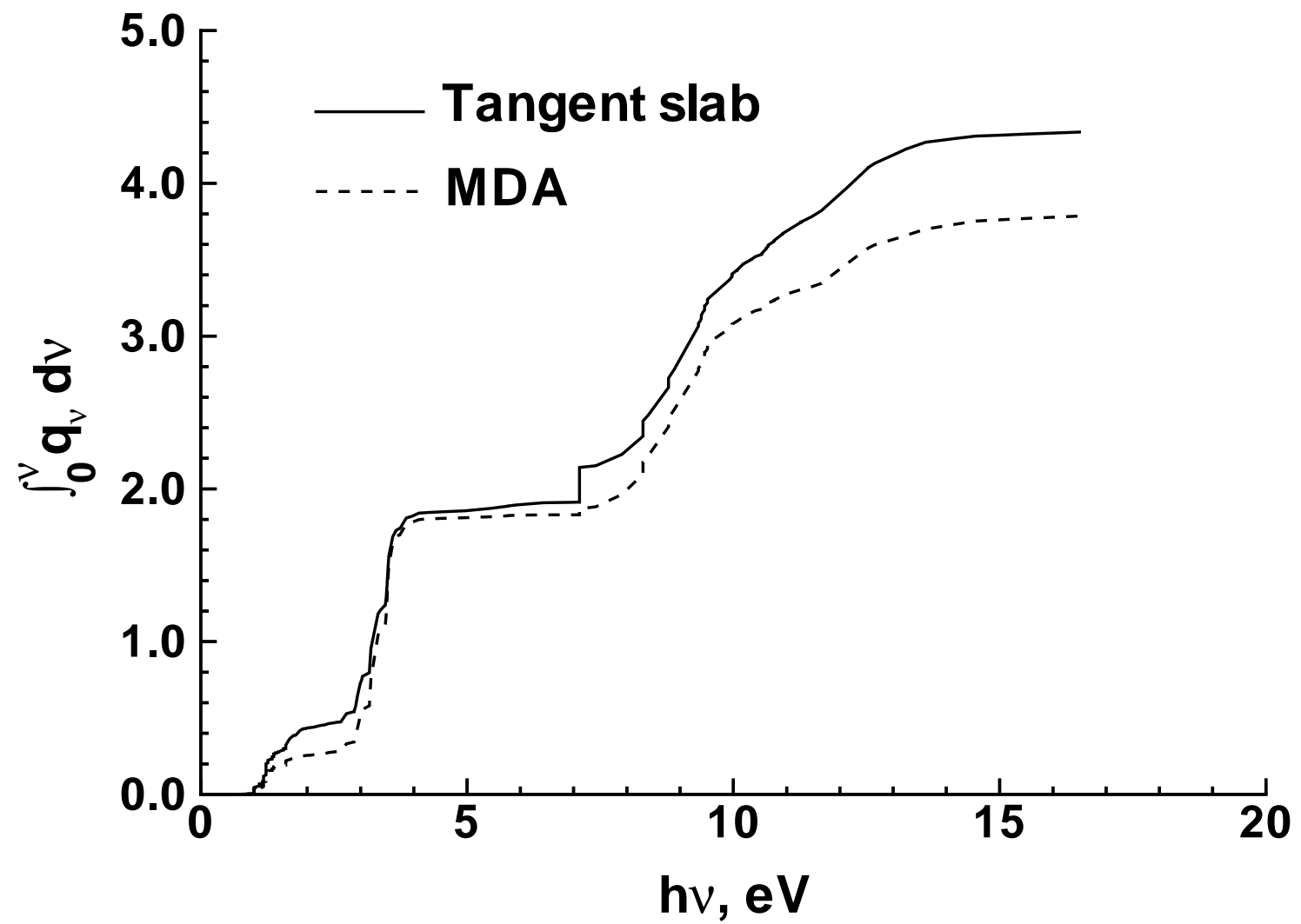

Figure 13: Spectral Distribution of Geosynchronous Return Wall Radiative Flux 